\title{
APLICAÇÃO DE PROGRAMAS DE ADUBAÇÃO FOLIAR COM SILÍCIO E POTÁSSIO ASSOCIADOS A FUNGICIDAS NO CONTROLE DE FERRUGEM ASIÁTICA NA CULTURA DA SOJA
}

\author{
PROGRAMS OF FOLIAR FERTILIZATION USING SILICON AND POTASSIUM \\ ASSOCIATED WITH FUNGICIDES TO CONTROL ASIAN RUST IN SOYBEANS
}

\begin{abstract}
Edson Ricardo de ANDRADE JUNIOR ${ }^{1}$; Daniel CASSETARI NETO ${ }^{2}$; Andréia Quixabeira MACAHADO ${ }^{3}$; Patrícia Maria Coury de Andrade VILELA ${ }^{4}$;

1. Engenheiro Agrônomo, M.Sc., Pesquisador do Departamento de Proteção de Plantas IMAmt, Cuiabá, MT, Brasil. edsonjunior@imamt.com.br; 2. Professor, Doutor, Departamento de Fitotecnia e Fitossanidade, Universidade Federal do Mato Grosso UFMT, Cuiabá, MT, Brasil; 3. Professora, M.Sc., UNIVAG, Várzea Grande, MT, Brasil; 4. Engenheira Agrônoma, M.Sc., Pesquisadora do IMAmt, Cuiabá, MT, Brasil.

RESUMO: O objetivo deste trabalho foi avaliar a eficiência de programas de adubação foliar com Si e K, associada a fungicidas no controle de ferrugem asiática na cultura da soja em condições de campo no Sul do Mato Grosso. O delineamento experimental foi de blocos ao acaso com 10 tratamentos e 4 repetições. As avaliações de severidade de ferrugem asiática foram realizadas nos estádios $R_{1}, R_{3}, R_{5.1}$ e $R_{5.3}$. Foi avaliada a porcentagem de desfolha (estádio $R_{6}$ ), massa de mil grãos e produtividade da cultura. $\mathrm{Na}$ avaliação final realizada em $\mathrm{R}_{5.3}$, a menor severidade da ferrugem asiática foi proporcionada pelos programas contemplados por epoxiconazole + piraclostrobin $\left(0,5 \mathrm{~L}^{-1}\right)$ em $\mathrm{R}_{1}$, tebuconazole + carbendazin $\left(0,5+0,5 \mathrm{~L} \mathrm{ha}^{-1}\right)$ em $\mathrm{R}_{3}$ e tebuconazole $\left(0,5 \mathrm{~L} \mathrm{ha}^{-1}\right)$ em $\mathrm{R}_{5.1}$ e por epoxiconazole + piraclostrobin $\left(0,5 \mathrm{~L} \mathrm{ha}^{-1}\right)$ em $\mathrm{R}_{1}$, adubação foliar $\left(0,5 \mathrm{~L} \mathrm{ha}^{-1}\right)$ em $\mathrm{R}_{3}$ e tebuconazole $\left(0,5 \mathrm{~L} \mathrm{ha}^{-1}\right)$ em $\mathrm{R}_{5.1}$. O programa epoxiconazole + piraclostrobin $\left(0,5 \mathrm{~L} \mathrm{ha}^{-1}\right)$ em $\mathrm{R}_{1}$, adubação foliar $\left(0,5 \mathrm{~L} \mathrm{ha}^{-1}\right)$ em $\mathrm{R}_{3}$ e tebuconazole $\left(0,5 \mathrm{~L} \mathrm{ha}^{-1}\right) \mathrm{em} \mathrm{R}_{5.1}$ proporcionou ganho em produtividade de $15,2 \mathbf{s c ~ h a}^{-1}$. Além de proporcionar uma redução na severidade da ferrugem asiática em soja, o uso associado de adubação foliar com Si e K e fungicidas promove aumento na produtividade da cultura.
\end{abstract}

PALAVRAS-CHAVE: Controle alternativo. Phakopsora pachyrhizi. Glycine max;

\section{INTRODUÇÃO}

A cultura da soja pode ser hospedeira de um complexo de agentes patogênicos com potencial limitante para a produtividade das lavouras. O uso de fungicidas tem sido a medida mais imediata no controle destes patógenos, visando proteger o potencial produtivo das lavouras, que pode ser reduzido em até $70 \%$ (EMBRAPA, 2006).

A antracnose, a mancha alvo e a partir do ano de 2001, a ferrugem asiática, consistem nas enfermidades mais importantes desse complexo de doenças, nas áreas de plantio do cerrado do CentroOeste e Nordeste do Brasil (ALMEIDA et al., 2005). Os sintomas iniciais da ferrugem são caracterizados por minúsculos pontos $(1-2 \mathrm{~mm}$ de diâmetro) mais escuros que o tecido sadio da folha, de uma coloração esverdeada a cinza-esverdeada. Devido ao hábito biotrófico (nutre-se do tecido vivo das plantas) do fungo, em cultivares suscetíveis, as células infectadas morrem somente após ter ocorrido abundante esporulação. Devido a isso, as lesões são facilmente visíveis no inicio da infecção (YORINORI et. al., 2002).

Segundo Zambolin et al. (2001), a nutrição das plantas determina, em grande parte, sua resistência ou suscetibilidade às doenças, suas estruturas histológicas ou morfológicas, as funções dos tecidos em reduzir a atividade patogênica, a virulência e habilidade do patógeno sobreviver. A deficiência dos nutrientes ao redor do ponto de infecção, tão necessários para sintetizar compostos químicos e barreiras físicas, pode resultar em suscetibilidade da planta as doenças.

Novas técnicas, como o uso de nutrição mineral, têm sido introduzidas no manejo integrado de doenças na cultura da soja. Os nutrientes são fatores ambientais que podem ser manipulados com relativa facilidade e usados como complemento no controle de doenças, uma vez que exercem importantes funções no metabolismo vegetal, influenciando tanto no crescimento e na produção, como nos mecanismos de defesa das plantas aos patógenos (CASSETARI et al., 2008).

O silício, apesar de não fazer parte da lista dos elementos essenciais as plantas, é um elemento cada vez mais utilizado na prática de manejo integrado de doenças. A aplicação de silicato de potássio na cultura da soja e milho tem proporcionado aumentos na produção, não só devido ao controle de doenças (principalmente a ferrugem), mas também pelo efeito na nutrição e 
metabolismo das plantas, devido ao aumento da eficiência fotossintética (RODRIGUES et al., 2007).

O objetivo deste trabalho foi avaliar a eficiência de programas contemplados com adubação foliar contendo $\mathrm{Si}$ e $\mathrm{K}$, associada a fungicidas no controle de ferrugem asiática (Phakopsora pachyrhizi) na cultura da soja em condições de campo.

\section{MATERIAL E MÉTODOS}

O experimento foi instalado na Fazenda Santo Antônio (Grupo Bom Futuro), no município de Campo Verde, Mato Grosso, coordenadas $15^{\circ} 18^{\prime} 34,24^{\prime \prime}$ S e $54^{\circ} 53^{\prime} 39,67^{\prime \prime O}$, altitude de $705 \mathrm{~m}$ em relação ao nível médio do mar, na safra 2007/2008. Foi utilizada a cultivar de soja Monsoy $9056 \mathrm{RR}$, semeada em linhas espaçadas de $0,45 \mathrm{~m}$ e densidade de plantio de 12 sementes $/ \mathrm{m}$, apresentando um estande final de 11,4 plantas $/ \mathrm{m}$ linear. $\mathrm{O}$ delineamento experimental foi o de blocos ao acaso com 10 tratamentos e 4 repetições (Tabela 1). Cada parcela foi constituída de 5 linhas de plantas de soja com 5 metros de comprimento, espaçadas de $0,45 \mathrm{~m}$, sendo considerada a área útil as 3 linhas centrais com 4 metros de comprimento.

Tabela 1: Programas de aplicação de fungicidas e adubos foliares com respectivos ingredientes ativos e doses avaliados no controle de Phakopsora pachyrhizi na cultura da soja. Campo Verde-MT, safra $2007 / 2008$

\begin{tabular}{|c|c|c|c|}
\hline \multirow[b]{2}{*}{ Programa } & \multicolumn{3}{|c|}{ Ingredientes Ativos (doses p.c. $\mathbf{L} \mathbf{h a}^{-\mathbf{1}}$ ) } \\
\hline & $1^{\text {a. }}$ Aplicação & $2^{\text {a. }}$ Aplicação & $3^{\text {a. }}$ Aplicação \\
\hline 1 & \multicolumn{3}{|c|}{ Testemunha - sem aplicação } \\
\hline 2 & $\begin{array}{l}\text { Epoxiconazole }+ \\
\text { Piraclostrobin }(0,5)\end{array}$ & $\begin{array}{l}\text { Tebuconazole }(0,5)+ \\
\text { Carbendazin }(0,5)\end{array}$ & Tebuconazole $(0,5)$ \\
\hline 3 & $\begin{array}{l}\text { Epoxiconazole }+ \\
\text { Piraclostrobin }(0,5)\end{array}$ & $\begin{array}{l}\text { Adubo Foliar - 35,1\% } \mathrm{P}_{2} \mathrm{O}_{5} \mathrm{e} \\
25,7 \% \mathrm{~K}_{2} \mathrm{O}(0,5)\end{array}$ & Tebuconazole $(0,5)$ \\
\hline 4 & $\begin{array}{l}\text { Adubo Foliar - 35,1\% } \mathrm{P}_{2} \mathrm{O}_{5} \\
\left.\text { e } 25,7 \% \mathrm{~K}_{2} \mathrm{O}(0,5)\right)\end{array}$ & $\begin{array}{l}\text { Epoxiconazole + Piraclostrobin } \\
(0,5)\end{array}$ & Tebuconazole $(0,5)$ \\
\hline 5 & $\begin{array}{l}\text { Adubo Foliar - 35,1\% } \mathrm{P}_{2} \mathrm{O}_{5} \\
\text { e } 25,7 \% \mathrm{~K}_{2} \mathrm{O}(0,5)\end{array}$ & $\begin{array}{l}\text { Tebuconazole }(0,5)+ \\
\text { Carbendazin }(0,5)\end{array}$ & Tebuconazole $(0,5)$ \\
\hline 6 & $\begin{array}{l}\text { Adubo Foliar - 35,1\% } \mathrm{P}_{2} \mathrm{O}_{5} \\
\text { e } 25,7 \% \mathrm{~K}_{2} \mathrm{O}(0,5)\end{array}$ & $\begin{array}{l}\text { Adubo Foliar - 35,1\% } \mathrm{P}_{2} \mathrm{O}_{5} \mathrm{e} \\
25,7 \% \mathrm{~K}_{2} \mathrm{O}(0,5)\end{array}$ & Tebuconazole $(0,5)$ \\
\hline 7 & $\begin{array}{l}\text { Adubo Foliar - 35,1\% } \mathrm{P}_{2} \mathrm{O}_{5} \\
\text { e } 25,7 \% \mathrm{~K}_{2} \mathrm{O}(0,5)\end{array}$ & $\begin{array}{l}\text { Tebuconazole }(0,5)+ \\
\text { Carbendazin }(0,5)\end{array}$ & $\begin{array}{l}\text { Adubo Foliar - } \\
35,1 \% \mathrm{P}_{2} \mathrm{O}_{5} \text { e } 25,7 \% \\
\mathrm{~K}_{2} \mathrm{O}(0,5)\end{array}$ \\
\hline 8 & $\begin{array}{l}\text { Adubo Foliar - 35,1\% } \mathrm{P}_{2} \mathrm{O}_{5} \\
\text { e } 25,7 \% \mathrm{~K}_{2} \mathrm{O}(0,5)\end{array}$ & $\begin{array}{l}\text { Adubo Foliar - 35, } 1 \% \mathrm{P}_{2} \mathrm{O}_{5} \mathrm{e} \\
\left.25,7 \% \mathrm{~K}_{2} \mathrm{O}(0,5)\right)\end{array}$ & $\begin{array}{l}\text { Tebuconazole }(0,5)+ \\
\text { Carbendazin }(0,5)\end{array}$ \\
\hline 9 & $\begin{array}{l}\text { Adubo Foliar - 35,1\% } \mathrm{P}_{2} \mathrm{O}_{5} \\
\text { e } 25,7 \% \mathrm{~K}_{2} \mathrm{O}(0,5)\end{array}$ & $\begin{array}{l}\text { Epoxiconazole + Piraclostrobin } \\
(0,5)\end{array}$ & $\begin{array}{l}\text { Adubo Foliar - } \\
35,1 \% \mathrm{P}_{2} \mathrm{O}_{5} \text { e } 25,7 \% \\
\mathrm{~K}_{2} \mathrm{O}(0,5)\end{array}$ \\
\hline 10 & $\begin{array}{l}\text { Adubo Foliar - 35,1\% } \mathrm{P}_{2} \mathrm{O}_{5} \\
\text { e } 25,7 \% \mathrm{~K}_{2} \mathrm{O}(0,5)\end{array}$ & $\begin{array}{l}\text { Adubo Foliar - 35,1\% } \mathrm{P}_{2} \mathrm{O}_{5} \mathrm{e} \\
25,7 \% \mathrm{~K}_{2} \mathrm{O}(0,5)\end{array}$ & $\begin{array}{l}\text { Adubo Foliar - } \\
35,1 \% \mathrm{P}_{2} \mathrm{O}_{5} \text { e } 25,7 \% \\
\mathrm{~K}_{2} \mathrm{O}(0,5)\end{array}$ \\
\hline
\end{tabular}

Foram realizadas três aplicações de cada programa em avaliação, em intervalos de 15 dias. As pulverizações foram efetuadas, utilizando-se um equipamento de pulverização costal de pressão constante $\left(\mathrm{CO}_{2}\right)$ com uma barra de 2 metros de comprimento com 4 bicos tipo cone vazio, operando com uma pressão de 50 psi e volume de calda de $200 \mathbf{L ~ h a}^{-1}$. As aplicações foram realizadas nos estádios fenológicos (descritos em EMBRAPA, 2008) $R_{1}, R_{3}$ e $R_{5.1}$ As condições climáticas durante as aplicações foram, com umidade relativa de $79 \%$, temperaturas mínima e máxima de $20,4^{\circ} \mathrm{C}$ e $28,2^{\circ} \mathrm{C}$, ventos variáveis entre 3,2 a $6,5 \mathrm{~km} / \mathrm{h}$ (brisa leve) sem ocorrência de precipitação.

As avaliações de severidade de ferrugem asiática foram realizadas nos estádios $R_{1}$ (no momento da $1^{a}$ aplicação dos tratamentos), $\mathrm{R}_{3}, \mathrm{R}_{5.1} \mathrm{e}$ $\mathrm{R}_{5.3} \quad$ (15 dias após a última aplicação dos tratamentos). Foi avaliada a severidade da doença (porcentagem de área foliar infectada), a Área Abaixo da Curva de Progresso da Doença (AACPD*) proposta por Campbell e Madden 
(1990), a porcentagem de desfolha (no estádio $\mathrm{R}_{6}$ ), a massa de mil grãos (MMG) e a produtividade (kg $\mathrm{ha}^{-1}$ ).

$$
* \mathrm{AACPD}=\Sigma\{(\mathrm{yi}+\mathrm{yi}+1) / 2\} \times\{\mathrm{ti}+1-\mathrm{ti}\}
$$

Onde:

$\mathrm{y}_{\mathrm{i}}=$ severidade da doença no tempo $\mathrm{t}$ severidade

$\mathrm{t}_{\mathrm{i}}=$ intervalo de tempo entre as avaliações da

Os dados foram submetidos à análise de variância e as médias dos tratamentos comparadas pelo teste de Tukey a $5 \%$ de probabilidade.

Os demais tratos culturais realizados: controle de pragas (lufenurom na dose de $0,3 \mathrm{~L} \mathrm{ha}^{-1}$, lambda-cialotrina na dose de $0,05 \mathrm{~L}^{-1} \mathrm{e}$ metamidofós na dose de $0,3 \mathrm{~L} \mathrm{ha}^{-1}$ ), plantas daninhas (2 aplicações de glifosato na dose de 2,5 L $\mathrm{ha}^{-1}$ ) e adubação (270 Kg ha ${ }^{-1}$ de 7-40-00 e $170 \mathrm{Kg}$ $\mathrm{ha}^{-1}$ de 0-0-60), foram os mesmos para todos os tratamentos seguindo o padrão da propriedade.

\section{RESULTADOS E DISCUSSÃO}

$\mathrm{Na}$ avaliação inicial de severidade, no estádio $\mathrm{R}_{1}$, não foi observada diferença estatística significativa entre os tratamentos, o que demonstra que a distribuição da ferrugem asiática na área do experimento encontrava-se homogênea, (Tabela 2).

$\mathrm{Na}$ avaliação realizada no estádio $\mathrm{R}_{3}$, os programas 2 e 3, com Epoxiconazole + Piraclostrobin $\left(0,5 \mathrm{~L} \mathrm{ha}^{-1}\right)$ na primeira aplicação se diferenciaram dos demais, sendo que esses programas receberam na primeira aplicação fungicida, já os demais programas, que receberam Adubo Foliar, contendo $24,13 \% \mathrm{~K}_{2} \mathrm{O}$ e $9,02 \% \mathrm{Si}$, não diferiram significativamente entre si, porém, diferiram significativamente do programa da testemunha, que não recebeu aplicação.

No estádio $\mathrm{R}_{5.1}$, observou-se que os menores índices de severidade foram alcançados novamente pelos programas 2 - Epoxiconazole + Piraclostrobin $\left(0,5 \mathrm{~L} \mathrm{ha}^{-1}\right)$ em $\mathrm{R}_{1} /$ Tebuconazole $\left(0,5 \mathrm{~L} \mathrm{ha}^{-1}\right)+$ Carbendazin $\left(0,5 \mathrm{~L} \mathrm{ha}^{-1}\right)$ em $\mathrm{R}_{3}$ e 3 - Epoxiconazole + Piraclostrobin $\left(0,5 \mathrm{~L} \mathrm{ha}^{-1}\right)$ em $\mathrm{R}_{1} /$ Adubo Foliar $\left(0,5 \mathrm{~L} \mathrm{ha}^{-1}\right)$ em $\mathrm{R}_{3}$. Os programas 5 - Adubo Foliar $\left(0,5 \mathrm{~L} \mathrm{ha}^{-1}\right)$ em $\mathrm{R}_{1} /$ Tebuconazole $\left(0,5 \mathrm{~L} \mathrm{ha}^{-1}\right)+$ Carbendazin $\left(0,5 \mathrm{~L} \mathrm{ha}^{-1}\right)$ em $\mathrm{R}_{3}$ e 7 - Adubo Foliar $\left(0,5 \mathrm{~L} \mathrm{ha}^{-1}\right)$ em $\mathrm{R}_{1} /$ Tebuconazole $\left(0,5 \mathrm{~L} \mathrm{ha}^{-1}\right)+$ Carbendazin $\left(0,5 \quad \mathrm{~L}^{-1}\right)$ em $\mathrm{R}_{3}$ não diferiram significativamente do programa 3 -Epoxiconazole + Piraclostrobin $\left(0,5 \mathrm{~L} \mathrm{ha}^{-1}\right)$ em $\mathrm{R}_{1} /$ Adubo Foliar $(0,5$ $\mathrm{L} \mathrm{ha}^{-1}$ ) em $\mathrm{R}_{3}$, sendo que estes receberam aplicação dos fungicidas Tebuconazole e Carbendazin na segunda aplicação.
Na avaliação final de severidade, no estádio $\mathrm{R}_{5.3}$, todos os programas diferiram significativamente da testemunha. O programa 2 Epoxiconazole + Piraclostrobin $\left(0,5 \mathrm{~L} \mathrm{ha}^{-1}\right) \mathrm{em}$ $\mathrm{R}_{1} /$ Tebuconazole $\left(0,5 \mathrm{~L} \mathrm{ha}^{-1}\right)+$ Carbendazin $(0,5 \mathrm{~L}$ $\mathrm{ha}^{-1}$ ) em $\mathrm{R}_{3} /$ Tebuconazole $(0,5) \mathrm{L} \mathrm{ha}^{-1}$ ) em $\mathrm{R}_{5.1}$, apresentou uma menor severidade da doença. Seguido pelo programa 3 - Epoxiconazole + Piraclostrobin (0,5 $\left.\mathrm{L} \mathrm{ha}^{-1}\right)$ em $\mathrm{R}_{1} /$ Adubo Foliar $(0,5$ $\left.\mathrm{L} \mathrm{ha}{ }^{-1}\right)$ em $\mathrm{R}_{3} /$ Tebuconazole $\left(0,5 \mathrm{~L} \mathrm{ha}^{-1}\right)$ em $\mathrm{R}_{5.1}$, que não diferiu do tratamento citado acima $\mathrm{e}$ recebeu uma aplicação de Adubo Foliar ( $2^{\mathrm{a}}$. Aplicação). O programa 5 - Adubo Foliar $\left(0,5 \mathrm{~L} \mathrm{ha}^{-}\right.$ $\left.{ }^{1}\right)$ em $\mathrm{R}_{1} /$ Tebuconazole $\left(0,5 \mathrm{~L} \mathrm{ha}^{-1}\right)+$ Carbendazin $\left(0,5 \mathrm{~L} \mathrm{ha}^{-1}\right)$ em $\mathrm{R}_{3} /$ Tebuconazole $\left(0,5 \mathrm{~L} \mathrm{ha}^{-1}\right)$ em $\mathrm{R}_{5.1}$ que também recebeu uma aplicação de Adubo Foliar ( ${ }^{\mathrm{a}}$. Aplicação) apresentou um baixo índice de severidade.

Com isso observou-se, com o programa 3 Epoxiconazole + Piraclostrobin $\left(0,5 \mathrm{~L} \mathrm{ha}^{-1}\right) \mathrm{em} \mathrm{R}_{1} /$ Adubo Foliar (0,5 $\left.\mathrm{L} \mathrm{ha}^{-1}\right)$ em $\mathrm{R}_{3}$ /Tebuconazole $(0,5$ $\mathrm{L} \mathrm{ha}^{-1}$ ) em $\mathrm{R}_{5.1}$ que poderíamos substituir uma aplicação de fungicida por uma de Adubo Foliar, na seqüência acima descrita.

Esses resultados estão de acordo com Reis e Guimarães (2008), que usando de silicato de potássio na dose de $4 \mathrm{~L} \mathrm{ha}^{-1}$, obteve eficiência semelhante ao fungicida epoxiconazole + piraclostrobin no controle da ferrugem e mancha de Phoma e Ascochyta no cafeeiro. Porém Roese et. al. (2009), estudando efeitos de indutores abióticos de resistência no controle de ferrugem da soja, observaram que o tratamento com cinco aplicações de silicato de potássio não diferenciou significativamente da testemunha. Aquino et.al. (2008) também não encontraram eficiência no silicato de potássio para o controle de ramularia no algodoeiro, obtendo índices de severidade e produtividade próximos da testemunha. Bezerra et. al. (2009), os quais estudando o efeito de silicato de potássio no desenvolvimento de Corynespora cassiicola in vitro observaram que o patógeno foi altamente sensível ao silicato de potássio, inibindo $100 \%$ do crescimento micelial, demonstrando que o produto possui ação sobre esse patógeno.

Com os dados da área abaixo da curva de progresso (Tabela 2), observou-se que os programas que proporcionaram menores áreas foram: 2 Epoxiconazole + Piraclostrobin $\left(0,5 \mathrm{~L} \mathrm{ha}^{-1}\right) \mathrm{em}$ $\mathrm{R}_{1} /$ Tebuconazole $\left(0,5 \mathrm{~L} \mathrm{ha}^{-1}\right)+$ Carbendazin $(0,5 \mathrm{~L}$ $\left.\mathrm{ha}^{-1}\right)$ em $\mathrm{R}_{3} /$ Tebuconazole $\left(0,5 \mathrm{~L} \mathrm{ha}^{-1}\right)$ em $\mathrm{R}_{5.1}$ (3 aplicações de fungicidas) e 3 - Epoxiconazole + Piraclostrobin $\left(0,5 \mathrm{~L} \mathrm{ha}^{-1}\right)$ em $\mathrm{R}_{1} /$ Adubo Foliar $(0,5$ $\left.\mathrm{L} \mathrm{ha}^{-1}\right)$ em $\mathrm{R}_{3} /$ Tebuconazole $\left(0,5 \mathrm{~L} \mathrm{ha}^{-1}\right)$ em $\mathrm{R}_{5.1}$ (duas aplicações de fungicida e uma de Adubo 
Foliar). Levando-se em consideração o complexo de doenças estudadas pode-se sugerir a substituição de uma aplicação de fungicida por adubo foliar, na sequiência acima descrita.

Os resultados de desfolha (porcentagem), avaliados no estádio $\mathrm{R}_{6}$, massa de mil grãos (MMG) e produtividade apenas confirmam os resultados anteriores, onde os programas 2 - Epoxiconazole + Piraclostrobin $\left(0,5 \mathrm{~L} \mathrm{ha}^{-1}\right)$ em $\mathrm{R}_{1} /$ Tebuconazole $(0,5$ $\left.\mathrm{L} \mathrm{ha}{ }^{-1}\right)+$ Carbendazin $\left(0,5 \mathrm{~L} \mathrm{ha}^{-1}\right)$ em $\mathrm{R}_{3} /$ Tebuconazole $\left(0,5 \quad \mathrm{~L} \quad \mathrm{ha}^{-1}\right)$ em $\mathrm{R}_{5.1}$ e 3 Epoxiconazole + Piraclostrobin $\left(0,5 \mathrm{~L} \mathrm{ha}^{-1}\right) \mathrm{em}$ $\mathrm{R}_{1}$ /Adubo foliar $\left(0,5 \mathrm{~L} \mathrm{ha}^{-1}\right)$ em $\mathrm{R}_{3} /$ Tebuconazole $\left(0,5 \mathrm{~L} \mathrm{ha}^{-1}\right)$ em $\mathrm{R}_{5.1}$ proporcionaram uma menor desfolha, uma maior massa de mil grão e uma maior produtividade, conseqüentemente com maiores ganhos quando comparados a testemunha, sendo de 17,7 e $15,2 \mathrm{sc} \mathrm{ha}^{-1}$ respectivamente (Tabela 3 ).

A produtividade obtida nas parcelas que receberam aplicação do programa 3 - Epoxiconazole + Piraclostrobin $\left(0,5 \mathrm{~L} \mathrm{ha}^{-1}\right)$ em $\mathrm{R}_{1} /$ Adubo foliar $\left(0,5 \mathrm{~L} \mathrm{ha}^{-1}\right)$ em $\mathrm{R}_{3} /$ Tebuconazole $\left(0,5 \mathrm{~L} \mathrm{ha}^{-1}\right)$ em $\mathrm{R}_{5.1}$ demonstrou que além de uma redução na severidade da ferrugem asiática, houve ganhos de produtividade quando substituiu-se uma aplicação de fungicida por uma de adubo foliar, contendo $35,1 \% \mathrm{P}_{2} \mathrm{O}_{5}$ e $25,7 \% \quad \mathrm{~K}_{2} \mathrm{O}$, conforme seqüência descrita acima, que além de um menor custo, não apresenta toxidade tanto para o ambiente (redução do impacto ambiental causado pelo uso de agroquímicos), quanto para o homem.

\section{CONCLUSÕES}

A adubação foliar com $\mathrm{Si}$ e $\mathrm{K}$, quando utilizada intercalada a aplicação de fungicidas, foi eficiente no controle de ferrugem asiática;

A adubação foliar com $\mathrm{Si}$ e $\mathrm{K}$, quando utilizada intercalada a aplicação de fungicidas, proporcionou ganhos de produtividade quando comparada a testemunha.

\section{AGRADECIMENTOS}

A Universidade Federal de Mato Grosso pelo ensino proporcionado no curso de mestrado em Agricultura Tropical e ao Instituto Mato-Grossense do Algodão, pelo apoio aos seus pesquisadores no desenvolvimento desse trabalho. 
Tabela 2. Severidade (\% de área foliar infectada) da ferrugem asiática na cultura da soja. Campo Verde-MT, safra 2007/2008

\begin{tabular}{|c|c|c|c|c|c|c|c|c|c|}
\hline \multirow{3}{*}{$\begin{array}{l}\left.\text { Programas (Programa de aplicação, I.A. doses ( } \mathbf{L} \mathbf{h a}^{\mathbf{- 1}}\right) \text { nas sequências de aplicação) } \\
\text { 1-Testemunha }\end{array}$} & \multicolumn{7}{|c|}{ Severidade } & \multirow{2}{*}{\multicolumn{2}{|c|}{$\begin{array}{c}\text { AACPD } \\
\text { Ferrugem }\end{array}$}} \\
\hline & \multirow{2}{*}{$\frac{\mathrm{R}_{1}}{4,1^{\mathrm{ns}}}$} & \multicolumn{2}{|c|}{$\mathrm{R}_{3}$} & \multicolumn{2}{|c|}{$\mathrm{R}_{5.1}$} & \multicolumn{2}{|c|}{$\mathrm{R}_{5.3}$} & & \\
\hline & & 52,1 & c & 72,6 & d & 100 & $\mathrm{e}$ & 2475,4 & d \\
\hline $\begin{array}{l}\text { 2- Epoxiconazole + Piraclostrobin }(0,5) / \text { Tebuconazole }(0,5)+\text { Carbendazin }(0,5) / \\
\text { Tebuconazole }(0,5)\end{array}$ & 3,6 & 14,8 & $\mathbf{a}$ & 20,4 & $\mathbf{a}$ & 30 & $\mathrm{a}$ & 727,1 & $\mathbf{a}$ \\
\hline $\begin{array}{l}\text { 3- Epoxiconazole + Piraclostrobin }(0,5) / \text { Adubo Foliar - 35, } 1 \% \mathrm{P}_{2} \mathrm{O}_{5} \text { e } 25,7 \% \mathrm{~K}_{2} \mathrm{O}(0,5) \\
\text { / Tebuconazole }(0,5)\end{array}$ & 3,4 & 13,1 & $\mathbf{a}$ & 29,8 & $\mathbf{a b}$ & 37,3 & $a b$ & 884,6 & $\mathbf{a}$ \\
\hline $\begin{array}{l}\text { 4- Adubo Foliar - } 35,1 \% \mathrm{P}_{2} \mathrm{O}_{5} \text { e } 25,7 \% \mathrm{~K}_{2} \mathrm{O}(0,5) / \text { Epoxiconazole + Piraclostrobin }(0,5) \\
\text { / Tebuconazole }(0,5)\end{array}$ & 4,8 & 32,5 & b & 62,3 & cd & 68 & $\mathrm{~cd}$ & 1835,8 & c \\
\hline $\begin{array}{l}\text { 5- Adubo Foliar - } 35,1 \% \mathrm{P}_{2} \mathrm{O}_{5} \text { e } 25,7 \% \mathrm{~K}_{2} \mathrm{O}(0,5) / \text { Tebuconazole }(0,5)+\text { Carbendazin } \\
(0,5) / \text { Tebuconazole }(0,5)\end{array}$ & 3,9 & 30,8 & b & 35,8 & b & 47,3 & $\mathrm{~b}$ & 1288,9 & b \\
\hline $\begin{array}{l}\text { 6- Adubo Foliar - 35,1\% } \mathrm{P}_{2} \mathrm{O}_{5} \text { e } 25,7 \% \mathrm{~K}_{2} \mathrm{O}(0,5) / \text { Adubo Foliar - 35, } 1 \% \mathrm{P}_{2} \mathrm{O}_{5} \text { e } 25,7 \% \\
\mathrm{~K}_{2} \mathrm{O}(0,5) / \text { Tebuconazole }(0,5)\end{array}$ & 3,9 & 31,8 & b & 54 & c & 69,8 & $\mathrm{~cd}$ & 1715,9 & c \\
\hline $\begin{array}{l}\text { 7- Adubo Foliar - 35,1\% } \mathrm{P}_{2} \mathrm{O}_{5} \text { e } 25,7 \% \mathrm{~K}_{2} \mathrm{O}(0,5) / \text { Tebuconazole }(0,5)+\text { Carbendazin } \\
(0,5) / \text { Adubo Foliar - 35,1\% } \mathrm{P}_{2} \mathrm{O}_{5} \text { e } 25,7 \% \mathrm{~K}_{2} \mathrm{O}(0,5)\end{array}$ & 4,4 & 32,1 & b & 43,8 & bc & 70 & $\mathrm{~cd}$ & 1582,9 & bc \\
\hline $\begin{array}{l}\text { 8- Adubo Foliar - } 35,1 \% \mathrm{P}_{2} \mathrm{O}_{5} \text { e } 25,7 \% \mathrm{~K}_{2} \mathrm{O}(0,5) / \text { Adubo Foliar - } 35,1 \% \mathrm{P}_{2} \mathrm{O}_{5} \text { e } 25,7 \% \\
\mathrm{~K}_{2} \mathrm{O}(0,5) / \text { Tebuconazole }(0,5)+\text { Carbendazin }(0,5)\end{array}$ & 2,6 & 32,5 & b & 64,3 & cd & 66,3 & c & 1836,6 & c \\
\hline $\begin{array}{l}\text { 9- Adubo Foliar - 35, } 1 \% \mathrm{P}_{2} \mathrm{O}_{5} \text { e } 25,7 \% \mathrm{~K}_{2} \mathrm{O}(0,5) \text { / Epoxiconazole + Piraclostrobin }(0,5) \\
\text { / Adubo Foliar - } 35,1 \% \mathrm{P}_{2} \mathrm{O}_{5} \text { e } 25,7 \% \mathrm{~K}_{2} \mathrm{O}(0,5)\end{array}$ & 4,3 & 30,8 & b & 60,5 & cd & 81,5 & d & 1877,8 & c \\
\hline $\begin{array}{l}\text { 10- Adubo Foliar - 35,1\% } \mathrm{P}_{2} \mathrm{O}_{5} \text { e } 25,7 \% \mathrm{~K}_{2} \mathrm{O}(0,5) / \text { Adubo Foliar - 35,1\% } \mathrm{P}_{2} \mathrm{O}_{5} \text { e } 25,7 \% \\
\mathrm{~K}_{2} \mathrm{O}(0,5) / \text { Adubo Foliar }-35,1 \% \mathrm{P}_{2} \mathrm{O}_{5} \text { e } 25,7 \% \mathrm{~K}_{2} \mathrm{O}(0,5)\end{array}$ & 2,6 & 32,5 & b & 56 & c & 81,3 & $\mathrm{~cd}$ & 1826,1 & c \\
\hline $\mathrm{CV}(\%)$ & 31,66 & & & & 76 & 9,4 & & 8,35 & \\
\hline
\end{tabular}

Médias seguidas de mesma letra na coluna não diferem entre si pelo teste de Tukey (5\%) ; ns = não significativo 
Tabela 3. Porcentagem de desfolha $\left(\mathrm{R}_{6}\right)$, Massa de mil grãos (MMG) e Produtividade média na cultura da soja. Campo Verde-MT, safra 2007/2008

\begin{tabular}{|c|c|c|c|c|}
\hline Programass (Programa de aplicação, I.A. com respectivas doses ( $\left.\mathbf{L} \mathbf{h a}^{-1}\right)$ nas sequências de aplicação) & $\begin{array}{l}\text { Desfolha } \\
(\%)\end{array}$ & $\begin{array}{c}\text { MMG } \\
(\mathrm{g})\end{array}$ & $\begin{array}{l}\text { Produtividade. } \\
\qquad\left(\mathbf{s c ~ h a} \mathbf{~ h}^{-1}\right)\end{array}$ & $\begin{array}{l}\text { Ganho } \\
\left(\mathbf{s c ~ h a}^{-1}\right)\end{array}$ \\
\hline 1-Testemunha & $100,0 \mathbf{c}$ & 91,2 a & $19,3 \quad \mathbf{a}$ & - \\
\hline 2- Epoxiconazole + Piraclostrobin $(0,5) /$ Tebuconazole $(0,5)+$ Carbendazin $(0,5) /$ Tebuconazole $(0,5)$ & $62,3 \mathbf{a}$ & $103,4 \mathbf{b}$ & 37,0 & 17,7 \\
\hline $\begin{array}{l}\text { 3- Epoxiconazole + Piraclostrobin }(0,5) / \text { Adubo Foliar - 35,1\% } \mathrm{P}_{2} \mathrm{O}_{5} \text { e } 25,7 \% \mathrm{~K}_{2} \mathrm{O}(0,5) / \\
\text { Tebuconazole }(0,5)\end{array}$ & $72,3 \mathbf{a b}$ & $108,1 \mathbf{b}$ & 34,5 & 15,2 \\
\hline $\begin{array}{l}\text { 4- Adubo Foliar - 35,1\% } \mathrm{P}_{2} \mathrm{O}_{5} \text { e } 25,7 \% \mathrm{~K}_{2} \mathrm{O}(0,5) / \text { Epoxiconazole + Piraclostrobin }(0,5) / \\
\text { Tebuconazole }(0,5)\end{array}$ & $81,5 \mathbf{b}$ & 90,0 a & 24,7 & 5,4 \\
\hline $\begin{array}{l}\text { 5- Adubo Foliar - 35,1\% } \mathrm{P}_{2} \mathrm{O}_{5} \text { e } 25,7 \% \mathrm{~K}_{2} \mathrm{O}(0,5) / \text { Tebuconazole }(0,5)+\text { Carbendazin }(0,5) / \\
\text { Tebuconazole }(0,5)\end{array}$ & $81,8 \mathbf{b}$ & $91,8 \mathbf{a b}$ & 24,5 & 5,2 \\
\hline $\begin{array}{l}\text { 6- Adubo Foliar - 35,1\% } \mathrm{P}_{2} \mathrm{O}_{5} \text { e } 25,7 \% \mathrm{~K}_{2} \mathrm{O}(0,5) / \text { Adubo Foliar - 35, } 1 \% \mathrm{P}_{2} \mathrm{O}_{5} \text { e } 25,7 \% \mathrm{~K}_{2} \mathrm{O}(0,5) / \\
\text { Tebuconazole }(0,5)\end{array}$ & 84,0 b & $92,5 \mathbf{a b}$ & 22,8 & 3,5 \\
\hline $\begin{array}{l}\text { 7- Adubo Foliar - 35,1\% } \mathrm{P}_{2} \mathrm{O}_{5} \text { e } 25,7 \% \mathrm{~K}_{2} \mathrm{O}(0,5) / \text { Tebuconazole }(0,5)+\text { Carbendazin }(0,5) / \text { Adubo } \\
\text { Foliar - } 35,1 \% \mathrm{P}_{2} \mathrm{O}_{5} \text { e } 25,7 \% \mathrm{~K}_{2} \mathrm{O}(0,5)\end{array}$ & $83,8 \mathbf{b}$ & $94,2 \mathbf{a b}$ & 24,2 & 4,9 \\
\hline $\begin{array}{l}\text { 8- Adubo Foliar - 35,1\% } \mathrm{P}_{2} \mathrm{O}_{5} \text { e } 25,7 \% \mathrm{~K}_{2} \mathrm{O}(0,5) / \text { Adubo Foliar - 35, } 1 \% \mathrm{P}_{2} \mathrm{O}_{5} \text { e } 25,7 \% \mathrm{~K}_{2} \mathrm{O}(0,5) / \\
\text { Tebuconazole }(0,5)+\text { Carbendazin }(0,5)\end{array}$ & $82,5 \mathbf{b}$ & $95,6 \mathbf{a b}$ & 28,3 & 9,0 \\
\hline $\begin{array}{l}\text { 9- Adubo Foliar - 35,1\% } \mathrm{P}_{2} \mathrm{O}_{5} \text { e } 25,7 \% \mathrm{~K}_{2} \mathrm{O}(0,5) / \text { Epoxiconazole + Piraclostrobin (0,5) / Adubo Foliar } \\
-35,1 \% \mathrm{P}_{2} \mathrm{O}_{5} \text { e } 25,7 \% \mathrm{~K}_{2} \mathrm{O}(0,5)\end{array}$ & 84,8 b & $90,5 \mathbf{a}$ & 25,0 & 5,7 \\
\hline $\begin{array}{l}\text { 10- Adubo Foliar - 35,1\% } \mathrm{P}_{2} \mathrm{O}_{5} \text { e } 25,7 \% \mathrm{~K}_{2} \mathrm{O}(0,5) / \text { Adubo Foliar - 35,1\% } \mathrm{P}_{2} \mathrm{O}_{5} \text { e } 25,7 \% \mathrm{~K}_{2} \mathrm{O}(0,5) / \\
\text { Adubo Foliar - } 35,1 \% \mathrm{P}_{2} \mathrm{O}_{5} \text { e } 25,7 \% \mathrm{~K}_{2} \mathrm{O}(0,5)\end{array}$ & 90,0 bc & $91,5 \mathbf{a}$ & 25,0 & 5,7 \\
\hline $\mathrm{CV}(\%)$ & 6,18 & 5,1 & 9,04 & - \\
\hline
\end{tabular}

Médias seguidas de mesma letra na coluna não diferem entre si pelo teste de Tukey (5\%). 


\begin{abstract}
The purpose of this study was to evaluate the effectiveness of foliar fertilization programs with Si and $\mathrm{K}$, combined with fungicides to control Asian rust in soybeans under field conditions in southern Mato Grosso. The experimental design was randomized blocks with 10 treatments and 4 replicates. Assessments of severity of rust were performed at stages $R_{1}, R_{3}, R_{5.1}$ and $R_{5.3}$. The percentage of defoliation (stage $R_{6}$ ), thousand grain weight and yield were evaluated. In the final evaluation, carried out in $\mathrm{R}_{5.3}$, the lower rust severity was provided by the programs covered by epoxiconazole + pyraclostrobin $(500 \mathrm{~mL} / \mathrm{ha})$ in $\mathrm{R}_{1}$, tebuconazole + carbendazin $(500+500 \mathrm{ml} / \mathrm{ha})$ and tebuconazole in $\mathrm{R}_{3}(500 \mathrm{~mL} / \mathrm{ha})$ in $\mathrm{R}_{5.1}$ and epoxiconazole + pyraclostrobin (500 mL / ha) in $\mathrm{R}_{1}$, foliar fertilization (500 $\left.\mathrm{mL} / \mathrm{ha}\right)$ in $\mathrm{R}_{3}$ and tebuconazole $(500 \mathrm{~mL} / \mathrm{ha})$ in $\mathrm{R}_{5.1}$. The program epoxiconazole + pyraclostrobin $(500 \mathrm{~mL} / \mathrm{ha})$ in $\mathrm{R}_{1}$, foliar fertilization (500 mL / ha) in $\mathrm{R}_{3}$ and tebuconazole $\left(500 \mathrm{~mL} / \mathrm{ha}\right.$ ) resulted in $\mathrm{R}_{5.1}$ productivity gain of 15.2 sacks / ha. Besides providing a reduction in the severity of soybean rust on soybeans, the combined use of foliar fertilization with $\mathrm{Si}$ and $\mathrm{K}$ and fungicides increases crop productivity.
\end{abstract}

KEYWORDS: Alternative control. Phakopsora pachyrhizi. Glycine max.

\title{
REFERÊNCIAS
}

ALMEIDA et al. - Doenças de Soja: Manual de Fitopatologia Vol. 2 - Doenças das Plantas Cultivadas. $4^{\text {a }}$. ed. Agronômica Ceres. São Paulo, SP. 2005. p. 569-588.

AQUINO, L. A.; BERGER, P. G.; RODRIGUES, F. A.; ZAMBOLIM, L.; OGOSHI, F.; MIRANDA, L. M.; LÉLIS, M. M. Controle alternativo de mancha de Ramularia do algodoeiro. Summa Phytopathology, Botucatu, v. 34, n. 2, p. 131-136. 2008.

BEZERRA, E. J. S.; SILVA, A. M.; BENTES, J. L. S. Efeito do Acibenzolar-S-metil, silicato de potássio e Ecolife no desenvolvimento de Corynespora cassiicola in vitro. Tropical Plant Pathology, Suplemento, vol. 34 p. 38. 2009.

CAMPBELL, C. L. \& MADDEN, L. V. Introduction to plant disease epidemiology. New York NY. Wiley. 1990.

CASSETARI, A. Q. M.; CASSETARI NETO, D.; ANDRADE JUNIOR, E. R. Soja - Equilíbrio perfeito, Revista Cultivar, Pelotas, Maio de 2008, pg. 18. 